\title{
The numerical simulation of ceramic composites failure at axial compression
}

\author{
P.V. Makarov, M.O. Eremin \\ Institute of Strength Physics and Material Science Siberian Branch of Russian Academy of Science, National Researching Tomsk \\ State University \\ bacardi@sibmail.com
}

\begin{abstract}
On the basis of the developed model of quasibrittle medium the brittle and quasibrittle failure of composite ceramic materials with zirconium dioxide matrix and various percentage of hardening particles of corundum are studied numerically. The theory of the damaged media is applied in the calculations. It is shown that failure process educes in 2 stages - a relatively slow quasistationary phase of accumulation of inelastic deformations and damages in all hierarchy of scales and superfast catastrophic phase - a blow-up regime when the failure process reaches the macro-scale and there is a macro-crack formation. The failure process in the proposed model assumes to be completed when the damage function reaches its maximum value and the strength of the composite evolve to zero.
\end{abstract}

KEYwORDS. Brittle and quasi-brittle failure; Quasistationary phase; Blow-up regime.

\section{INTRODUCTION}

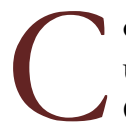

onstructional ceramic composites have received a wide practical application in the industry because of their high unit strength, the raised toughness, hardness, crack stability, high resistivity to fatigue breakdown etc. Constructional ceramics on the basis of strong oxides of various metals (zirconium, aluminum, etc.) are capable to resist the intensive mechanical loads effectively. However the brittle properties of ceramic composites, their rather low stability to shock loads strongly confine the ranges of their application. Studying the mechanisms and features of brittle and quasibrittle failure of ceramic materials is one of the most urgent problems of modern fracture mechanics.

According to the approach of physical mesomechanics [1] and ideas of paper [2] the loaded solid is the nonlinear dynamic system which evolution in fields of operating forces completely corresponds to the fundamental features of evolution of nonlinear dynamic systems.

In the present paper the basic attention is given to the character of failure of quasibrittle solids and media - the presence of two stages of failure: rather slow quasistationary and superfast catastrophic stage - the blow-up regime according to the S.P. Kurdyumov terminology [3] as it is the fundamental feature of evolution of the nonlinear dynamic systems possessing the self-organized criticality [4].

One of the central problems of solid mechanics is a problem of the formulation of the fracture criteria or conditions when the crack formation starts. Many papers are dedicated to the problems of crack-growing in non-homogeneous media, such as rocks [19], polycrystalline materials or ceramics and ceramic composites [18]. For example, in paper [21] the equation of state is built on the basis of crack interaction and crack density accounting and strain rate dependency and used for simulation of macroscopic stress-strain curves. Such approach gives good qualitative and quantitative results in calculation of the degradation stage in stress-strain curves, strain rate dependency and defects density evolution. However 
in the present paper the main accent is made on studying the transition from the quasistationary phase of media evolution to the blow-up regime which is assumed as local failure at crack formation which applies to be the new vision of the failure process.

In the present paper these problem also dares on the basis of ideas of the mathematical theory of evolution of loaded solids and media [2].

According to traditional ideas of fracture mechanics a local fracture in solids occurs when a maximum load is reached. All experience of application of this approach to the problem of limiting design has shown its comprehensible working capacity and correctness for many practical problems. However we can tell nothing about the failure process especially about its forecast. If a certain constant or changing load is enclosed to solid it is only possible to calculate the conforming stress-strain state and to answer a question whether a maximum load is reached or not somewhere. Such answers appear useful and sufficient in a number of important engineer cases but to tell something about the mechanisms and scenarios of failure locus formation is impossible.

The fundamental law of fracture of any materials has been investigated in the 70s of the XX century: the final failure (not only fatigue but any) precedes more or less significant preparatory stage. For example for the silicate glasses which failure was considered as instant the speed of crack propagation in the beginning of failure has appeared in thousands times less than at the final stage [5] and this is with the fact that the whole failure process takes some ms.

Rapid development of ideas and methods of nonlinear dynamics at the same years and the next decades have allowed the group of S.P. Kurdyumov to formulate the new concept of superfast catastrophic stages of evolution of nonlinear systems - the blow-up regimes [3] and both analytically and numerically to study the kinds and features of these regimes.

These ideas and the qualitative results obtained on their basis are the key-ideas for understanding the failure process. In the considered case of brittle or quasibrittle failure (and also the failure of any materials and constructions, plastic metals, brittle concrete, rocks, geomedia etc.) the preparatory process of accumulation of inelastic deformations and damages in brittle media is localized in certain areas. This preparatory quasistationary stage because of the self-organized criticality of solid as nonlinear dynamic system passes sooner or later in the superfast catastrophic stage - blow-up regime by S.P. Kurdyumov [2, 4, 7]. It is clear that any failure forecast basically is not possible without studying the features of development of these stages and conditions of transferring of one stage of sustainable development of failure to unstable superfast regime.

\section{MATHEMATICAL STATEMENT OF A PROBLEM. MODEL OF QUASI-BRITTLE MEDIUM}

ccording to the evolutionary concept of the description of inelastic deforming and the subsequent failure processes [2, 4, 6-9] the full set of equations includes:

1. Fundamental conservation laws:

$\begin{array}{ll}- \text { Mass } & \frac{d \rho}{d t}+\rho \operatorname{div} \bar{v}=0 \\ - \text { Impulse } & \rho \frac{d v_{i}}{d t}=\frac{\partial \sigma_{i j}}{\partial x^{j}}+\rho F_{i} \\ - \text { Energy } & \frac{d E}{d t}=\frac{1}{\rho} \sigma_{i j} \frac{d \varepsilon_{i j}}{d t}\end{array}$

where $\rho$ is the material density, $v_{i}$ is the $i$-component of the speed vector, $F_{i}$ is the $i$-component of the massive force, is energy, $t$ is time.

2. The evolutionary equations of the first group which have been written down in the relaxation form in which increments of stresses $\Delta \sigma_{i j}=\dot{\sigma} \Delta t$ are proportional to increments of total deformations $\dot{\varepsilon}_{\mathrm{ij}}^{\mathrm{T}}$ and relax proportionally to the development of inelastic deformation $\dot{\varepsilon}_{i j}^{p}$. The procedure of stresses reduction to the instant limiting surface means the instant stress relaxation on each time layer to some dynamic equilibrium state defined both by relaxation and the rate of strength and elastic parameters of medium degradation. At $\dot{\varepsilon}_{i j}^{P}>\dot{\varepsilon}_{i j}^{T} \quad \Delta \sigma_{i j}<0$, there is a relaxation, at $\dot{\varepsilon}_{i j}^{P}<\dot{\varepsilon}_{i j}^{T} \quad \Delta \sigma_{i j}>0$ and stresses steepen:

$$
\dot{\sigma}_{i j}=\lambda\left(\dot{\theta}^{T}-\dot{\theta}^{P}\right) \delta_{i j}+2 \mu\left(\dot{\varepsilon}_{i j}^{T}-\dot{\varepsilon}_{i j}^{P}\right)
$$




$$
\begin{aligned}
& \sigma_{i j}=\left(-P \delta_{i j}+S_{i j}\right) \\
& \dot{P}=-K \frac{\dot{V}}{V} \\
& \frac{D S_{i j}}{D t}=2 \mu\left(\dot{\varepsilon}_{i j}-\frac{1}{3} \dot{\theta} \delta_{i j}\right) \\
& \frac{D S_{i j}}{D t}=\dot{S}_{i j}-S_{i k} \dot{\omega}_{j k}-S_{j k} \dot{\omega}_{i k} \\
& \dot{\varepsilon}_{i j}^{T}=\frac{1}{2}\left(\frac{\partial v_{i}}{\partial x^{j}}+\frac{\partial v_{j}}{\partial x^{i}}\right) \\
& \dot{\omega}_{i j}=\frac{1}{2}\left(\frac{\partial v_{i}}{\partial x^{j}}-\frac{\partial v_{j}}{\partial x^{i}}\right)
\end{aligned}
$$

$\dot{\theta}$ is the rate of volumetric strain, $\lambda, \mu$ are the Lame constants, $K$ is the bulk modulus, $P$ is pressure, $\sigma_{i j}$ are the components of deviator stress tensor, $\frac{D}{D t}$ is the co-rotation derivative of Yauman considering the rotation of the medium elements at deforming, $\dot{\omega}_{i j}$ are the components of the rotation strain rate tensor.

3. The problem of the evolutionary equations of the second group is the definitions of rates of inelastic deformations in the Eq. (4). Generally it is the kinetic equations setting the rates of inelastic deformations and providing the relaxation of elastic stresses in (4). In the present paper the components of rates of inelastic deformations tensor are identified according to the theory of plasticity and instant stress relaxation on each time layer.

The limiting surface of stresses is written down in the form of Mises-Schleicher that allows to satisfy the requirement of generalization of plasticity and brittle failure conditions: the form of a limiting surface and its properties are completely defined by three parameters of a stressed state - octahedral normal stress octahedral shear stress $\sigma_{\text {oct }}$ and a kind of the stressed state $\mu_{\sigma}$ (parameter of Lode-Nadai)

$$
f=\frac{\alpha}{3} J_{1}\left(\sigma_{i j}\right)+J_{2}^{1 / 2}\left(\sigma_{i j}\right)-Y
$$

where $f$ is the yield surface and $J_{1}, J_{2}$ are the first, the second invariants of the stress tensor and $Y$ - current strength. Eq. (11) is a generalization of plasticity criterion of Coulomb-More. The model of Drucker-Prager-Nikolaevskiy with nonassociated flow law is taken as basis allowing describing the dilatation and internal friction processes independently. In the case of non-associated flow law the plastic potential $g\left(\sigma_{i j}\right)$ does not coincide with a function of plasticity and for a limiting surface (11) is written as follows [10]:

$$
g\left(\sigma_{i j}\right)=J_{2}+\frac{\Lambda}{3} J_{1}\left(2 Y-\frac{\alpha}{3} J_{1}\right)+\text { const }
$$

Components of rates of inelastic deformations tensor will be defined as follows:

$$
\dot{\varepsilon}_{i j}^{p}=\dot{\lambda} \frac{\partial g}{\partial \sigma_{i j}}=\left(s_{i j}+\frac{2}{3} \Lambda\left(Y-\frac{\alpha}{3} I_{1}\right) \delta_{i j}\right) \dot{\lambda}
$$

where $\dot{\lambda}$ is the plasticity multiplier in the theory of plasticity.

$$
\dot{I}_{1}^{P}=2 \Lambda\left(\dot{I}_{2}^{P}\right)^{1 / 2}
$$

That allows to establish the connection between volumetric $\dot{I}_{1}^{P}$ and shear $\dot{I}_{2}^{P}$ components of inelastic strain (14) [7] where $\Lambda$ is a speed of dilatation. However the model is not bound yet to the kind of stressed state. That dependence will be defined within the function of damages accumulation. 
4. Failure in the educed approach is considered as a process of avalanche degradation of material strength to zero at macro-cracking during the superfast catastrophic stage of stress-strain state evolution which is the closing stage of prefailure. However the medium remains consolidated macroscopically hence all the equations of inelastic deforming (1) $\div$ (14) are fair. There is no need to introduce the strength parameters defining the "limiting" state of material into model. According to the ideas of the present paper the "limiting" condition should be formed in the loaded medium during the process of inelastic deformations and damages accumulation. It is necessary to set the initial strength of the material .

According to the classic ideas of the failure kinetic concept (N.S. Zhurkov, A.V. Stepanov, R. Bekker, Ja.I. Frenkel and others) [11-15] to lead an ideal crystal to a state of local shear it is necessary to make a work proportional to the difference of free energies $\mathrm{F}$ of an ideal crystal and a crystal in current state $A(\sigma) \sim V \frac{\sigma_{0}^{2}-\sigma^{2}}{2 \mu}\left(\mathrm{V}\right.$ - volume, $\sigma_{0}$ - the value of theoretical strength, $\sigma$ is the current stress).

Orowan modified this idea and put the critical increment of energy depending only on the size of plastic (inelastic) deformation [16] $F(\sigma) \sim \mathrm{h}^{2} \mathrm{~V} \frac{\varepsilon_{\mathrm{p}}^{2}}{2 \mu}$, where $h$ is the strain hardening parameter, $\varepsilon_{\mathrm{p}}$ is the accumulated inelastic strain.

We use this idea and put the function of medium degradation $D=D\left(t, \varepsilon_{p}, \mu_{\sigma}\right)$ in the form of dependence on inelastic deformation accumulated by medium $\varepsilon_{p}=\varepsilon_{c u r}-\varepsilon_{0}$ and the kind of stressed state:

$$
\begin{aligned}
& D=\int_{t_{0}}^{t} \frac{\left(\varepsilon_{\mathrm{cur}}-\varepsilon_{0}\right)^{2} \mathrm{dt}}{\varepsilon_{*}^{2} t_{*}} \\
& \varepsilon_{*}=\varepsilon_{0 *}\left(1+\mu_{\sigma}\right)^{n}, \quad \mathrm{Y}=\mathrm{Y}_{0}(1-\mathrm{D}), \mathrm{D} \leq 1 \\
& \mu_{\sigma}=2 \frac{S_{2}-S_{3}}{S_{1}-S_{3}}-1
\end{aligned}
$$

$\varepsilon_{\text {cur }}$ is the current mean of total deformation intensity, $\varepsilon_{0}$ is the initial deformation when the damage accumulation begins. $\varepsilon_{0}$ is different for areas of compression and tension and makes $0.2-0.5$ from the elasticity limit depending on a solved problem. Such approach allows accumulating the damages at macroscopically elastic stage of deforming. Rates of damage accumulation for local tension-shift areas where $\mu_{\sigma}<0$ are essentially bigger than in compression-shift areas where $\mu_{\sigma}>0$. This process is controlled by the parameter $\varepsilon_{*}=\varepsilon_{*}\left(\mu_{\sigma}\right)$ in (15). Thus the medium response (its current strength) is formed during loading. Hence the strength and elastic parameters will degrade essentially faster in those areas (particles) of medium where the Lode-Nadai parameter $\mu_{\sigma}<0$ that corresponds to tension-shift areas. This response depends also on the loading history. Changing the deforming regime from tension-shear to compression-shear might mean the transition to another scenario of evolution and regeneration of the medium properties. $\varepsilon_{0} *$ is the model parameter, $t *$ makes sense of the characteristic time of the process, $S_{1}, S_{2}, S_{3}$ - are the main deviatric stresses.

Calculations were made in $2 \mathrm{D}$ under the condition of a plane deformation and $3 \mathrm{D}$ with the scheme of the second order of accuracy described in detail in paper [17].

\section{THE NUMERICAL SIMULATION RESULTS OF BRITTLE AND QUASI-BRITTLE FAILURE OF COMPOSITE CERAMIC MATERIALS}

$\sqrt{1}$ he model specimens of ceramic composites with zirconium dioxide $\mathrm{ZrO}_{2}$ matrix and various content of hardening particles $\left(15 \%\right.$ (a) and $40 \%$ (b)) of corundum $\mathrm{Al}_{2} \mathrm{O}_{3}$ are represented on Fig. 1. The content of the second phase was chosen as $15 \%$ and $40 \%$ to study the qualitative changes in the mechanical behavior of the composites. The presented structural models were developed on the basis of the well-known typical quasi-homogeneous distribution of the hardening particles within the matrix. In paper [22] the experimental study of uniaxial compression of porous ceramic on the base of zirconium dioxide was carried out and it's structure and phase content were also studied. Using the observations of structure from several papers of the author of paper [22] we developed the model of ceramic 
composites presented on the Fig. 1. The uniaxial compression of specimens was carried out in the calculations. The dimensions of the specimens are $100 \times 140 \mu \mathrm{m}$.

In paper [18] it has been shown that in loaded ceramic composites there are local areas of tension stresses on the interphase borders. Formation of mesocracks occurs in these areas of tension stresses. We will show that failure of composites in the majority of cases occurs in tension areas. It is caused by two reasons: 1) the presence of structural heterogeneities always leads to the formation of local tension areas in composite; 2) strength of quasibrittle materials at tension is essentially low than at compression. Rates of damages accumulation in tension areas are also essentially bigger.

In Tab. 1 the physical-mechanical properties of the materials compounding the composite are presented.

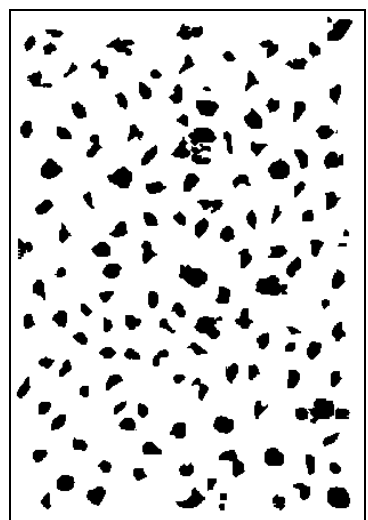

(a)

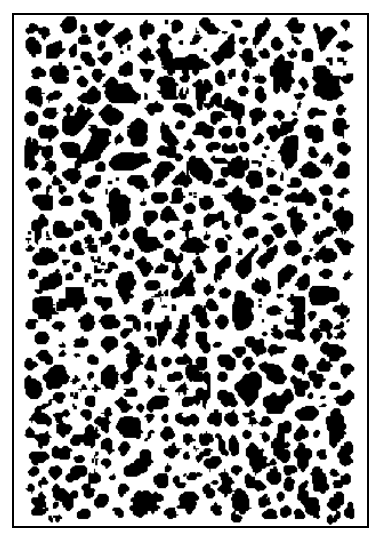

(b)

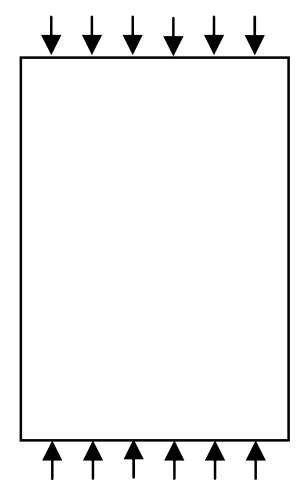

(c)

Figure 1: The model specimens of composites with $15 \%$ (a) and $40 \%$ (b) content of the hardening particles and the principle loading scheme (c).

\begin{tabular}{lcccccc}
\hline Parameter & $\begin{array}{c}\text { Material } \\
\text { density, } \\
\mathrm{kg} / \mathrm{m}^{3}\end{array}$ & $\begin{array}{c}\text { Bulk modulus, } \\
\mathrm{MPa}\end{array}$ & $\begin{array}{c}\text { Shear modulus, } \\
\mathrm{MPa}\end{array}$ & Strength, MPa & $\begin{array}{c}\Lambda \\
\text { speed of } \\
\text { dilatation }\end{array}$ & $\begin{array}{c}\alpha, \text { internal } \\
\text { friction } \\
\text { coefficient }\end{array}$ \\
$\mathrm{ZrO}_{2}$ & 5700 & $1.433 \cdot 10^{5}$ & $0.6615 \cdot 10^{5}$ & 2100 & 0.22 & 0.62 \\
$\mathrm{Al}_{2} \mathrm{O}_{3}$ & 3984 & $3.46 \cdot 10^{5}$ & $1.6 \cdot 10^{5}$ & 3740 & 0.12 & 0.6 \\
\hline
\end{tabular}

Table 1: The physical-mechanical properties of the materials compounding the composite.

\section{The macroscopic behavior}

The $\sigma-\varepsilon$ diagrams for composite specimens are shown under various loading conditions on Fig. 2 - ideal sliding (Fig. 2a) and friction (Fig. 2b) on the loading border.

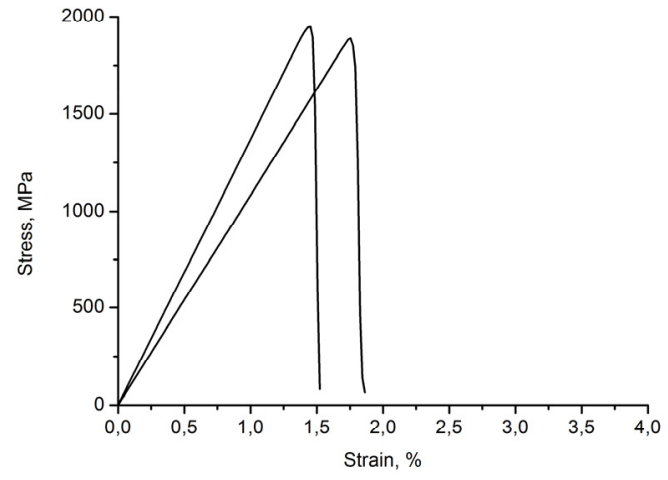

(a)

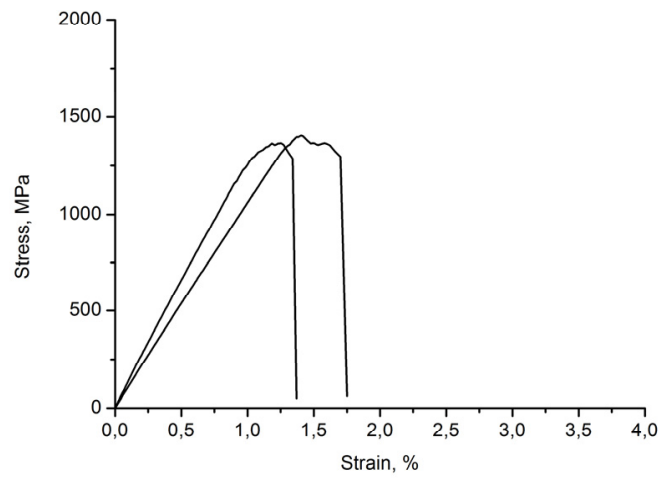

(b)

Figure 2: The $\sigma-\varepsilon$ diagrams in case of: ideal sliding (a), friction (b) on the loading border. 
The general for two variants of loading is the strength evolution of composites in the blow-up regime at final stage of deforming. Steady slow accumulation of inelastic deformations and damages at lower structural levels than considered macrolevel is replaced by the stage of unstable avalanche accumulation of damages (the blow-up regime) which occupies the tenth lobes of macrodeformation. It means that there is a localization of failure process in time. During the blow-up regime the global loss of stability, strength and elastic moduli of composites degradation to zero occur very quickly.

In the case of ideal sliding on the loading border (Fig. 2a) the brittle failure of specimens occurs that is the sharp break on the $\sigma-\varepsilon$ diagram and the system evolution is observed in the blow-up regime. The stage of the linear stress growth is followed by the blow-up regime with the global strength degradation. The feature of the deformation response in the case of friction on the loading border (Fig. 2b) is the presence of the stage of inelastic deforming of specimens because of the compression-shear areas formation near the friction border that sharply reduces the rate of damages accumulation. This process detains the phase of the blow-up regime and the global degradation of physical-mechanical properties of medium. Thus the stage of inelastic deformation occupies some $\%$ of macrodeformation.

Other scenario educes in the case of a lateral pressure (Fig. 3). Constraint of the deformation leads to tightening of the catastrophic phase of evolution of the system. The strength resource is spent gradually in this case, the sequence of relaxations and growths of stresses is observed. The similar situation is observed in geomedia at so-called "slow" earthquakes when the features of constraint of deformation lead to the tightening of the local failure process.

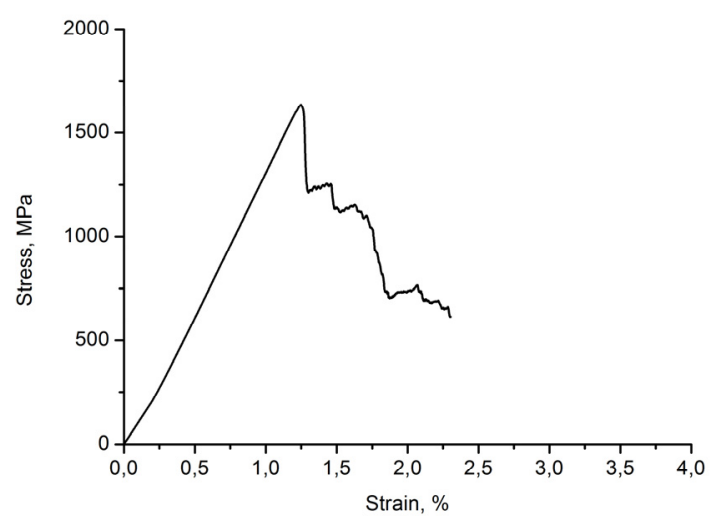

Figure 3: The $\sigma-\varepsilon$ diagram in the case of the lateral pressure.

\section{The behavior on the meso-scale}

The part of specimen area reflecting the kind of the stressed state in a composite defined by the Lode-Nadai parameter is represented on Fig. 4. First of all the formation of a strip of the localized failure occurs in a narrow regions of normal tension stresses $\mu_{\sigma} \rightarrow-1$.

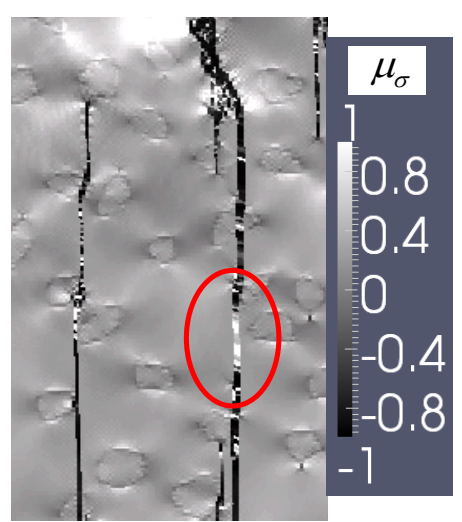

Figure 4: The part of specimen area reflecting the kind of the stressed state in the composite defined by the Lode-Nadai parameter $\mu_{\sigma}$.

Besides in the generated mesocrack in the noted area the stressed state was replaced with compression-shears that means transferring to the compaction mode at the steepening of the deformation constraint due to the presence of the hardening 
particles. At the same time in the generated mesocrack areas neighboring with the compaction zone are in dilatation regime. This process of failure delay in matrix shows one more positive role of the hardening particles.

On Fig. $5-8$ the calculated patterns of inelastic deformations in composites for three consecutive times $\left(t_{1}=4000 \mu s, t_{2}=6000 \mu s, t_{3}=8000 \mu s\right)$, the stressed state (by the Lode-Nadai parameter), damage function are presented at 2 variants of boundary conditions.

In all presented cases the incipient states of specimens deforming accompanied with a vertical crack growing that also show the experiments [20].

Comparison of failure patterns of composites with various percentage of hardening particles shows the distinction in mechanisms of their destruction. In the composite with $40 \%$ content of corundum the great number of hardening particles simultaneously creates the possibility for formation of the of local tension stresses areas on the phase border where the degradation of physical-mechanical properties of medium descends much faster. But also interferes with the macrocrack growing. On the pre-failure stage of the composite with $40 \%$ content of corundum the significant number of mesocrack is generated and this is more power-intensive process than formation of the small number of long cracks within the composite with $15 \%$ content of hardening particles (Fig. 5, 7).

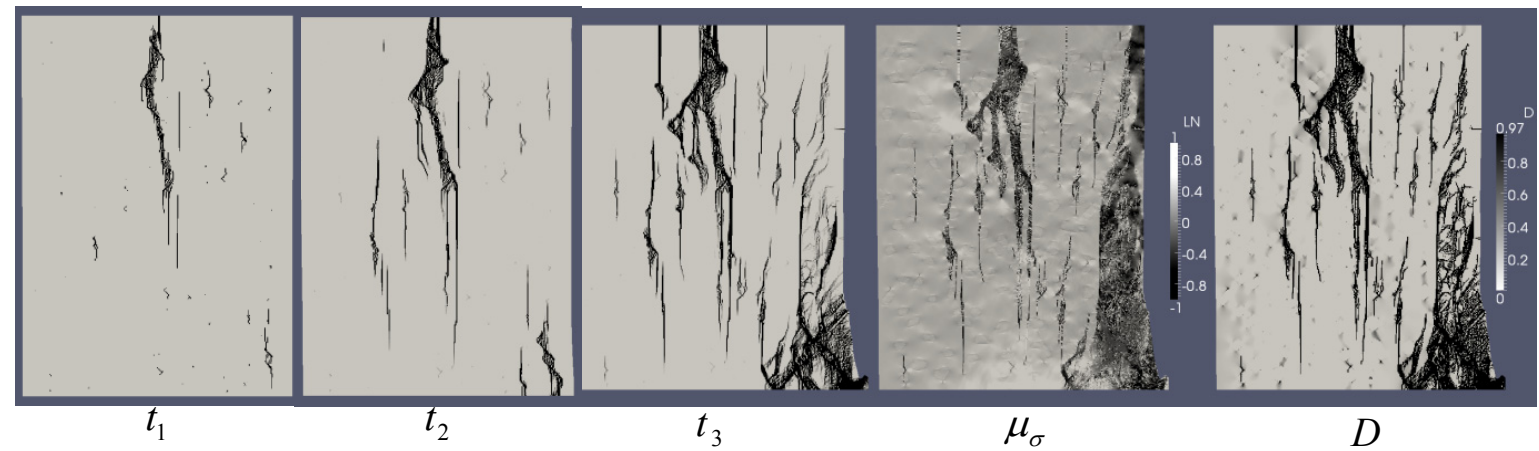

Figure 5. Simulation patterns: inelastic deformations (for three consecutive times), the stressed state $\left(\mu_{\sigma}\right)$, damage function $(D)$ in the composite specimen with $15 \%$ content of hardening particles in the case of ideal sliding on the loading border.

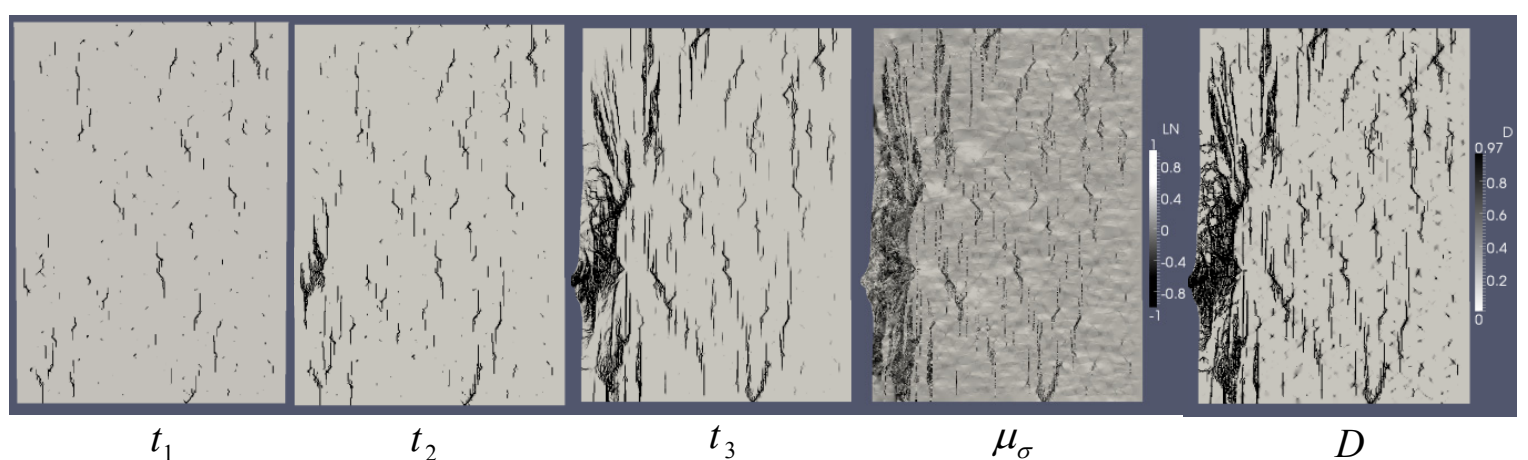

Figure 6: Simulation patterns: inelastic deformations (for three consecutive times), the stressed state $\left(\mu_{\sigma}\right)$, damage function $(D)$ in the composite specimen with $40 \%$ content of hardening particles in the case of ideal sliding on the loading border.

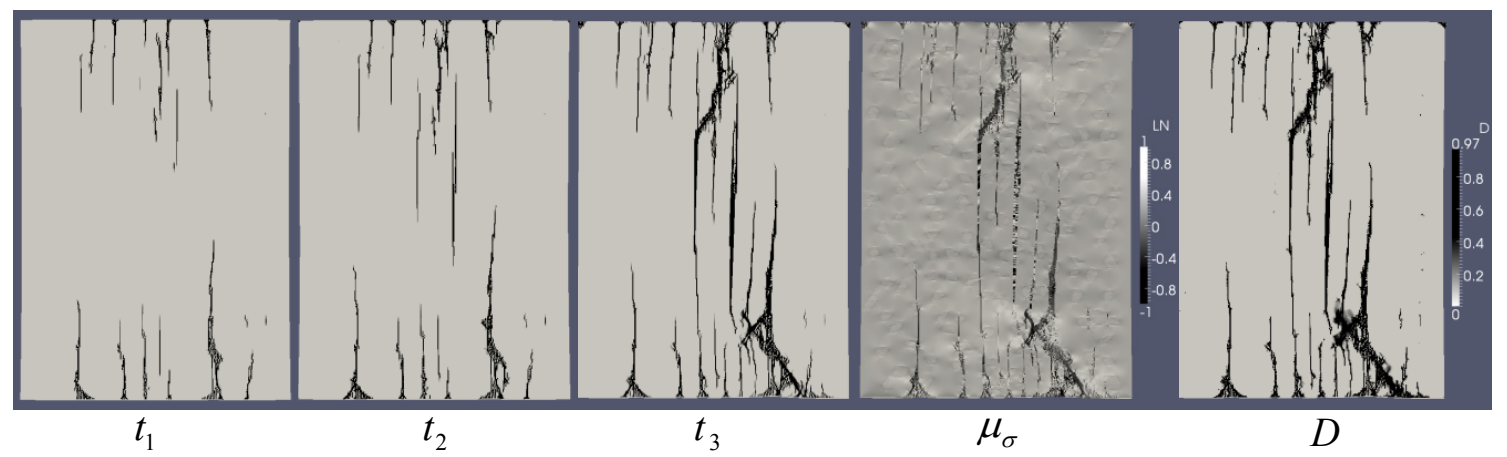

Figure 7: Simulation patterns: inelastic deformations (for three consecutive times), the stressed state $\left(\mu_{\sigma}\right)$, damage function $(D)$ in the composite specimen with $15 \%$ content of hardening particles in the case of friction on the loading border. 


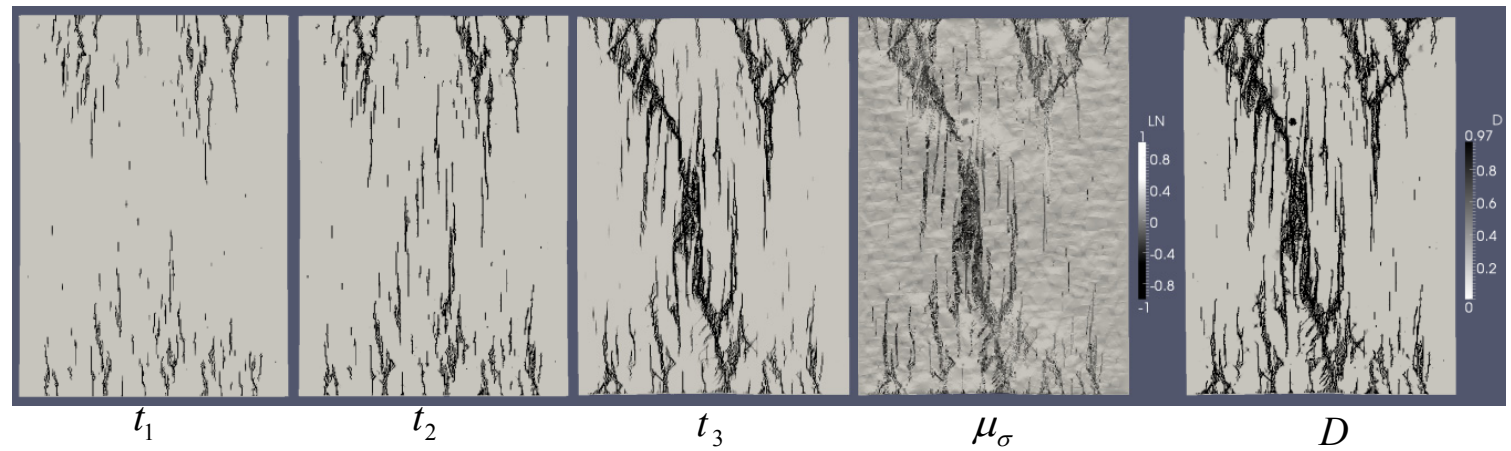

Figure 8: Simulation patterns: inelastic deformations (for three consecutive times), the stressed state $\left(\mu_{\sigma}\right)$, damage function $(D)$ in the composite specimen with $40 \%$ content of hardening particles in the case of friction on the loading border.

Dynamics of crack growth in such composites (Fig. 6, 8) for three consecutive times shows that during the pre-failure stage there is a steady accumulation of inelastic deformations and damages in the whole considered volume of composites. The catastrophic superfast phase of system evolution - the blow-up regime occurs when the process of inelastic deformations and damages accumulation reaches the macrolevel. It is anticipated by the quasistationary preparation phase which is expressed in the formation of percolation net of mesocracks. At final stage of deforming there is a confluence of the generated mesocracks in main macrocrack.

Other situation is observed in the composite with $15 \%$ content of hardening particles. Propagation of the crack fastigium generated on the interphase border is stopped by the smaller quantity of hardening particles that is the growing crack approximately three times rarely encounters the resistance of hardening particles. Thus the average length of an individual mesocrack for the composite with 15\% content of corundum exceeds the similar length of an individual mesocrack for the composite with $40 \%$ content of corundum in 1.5 - 2 times. Such distinction at mesolevel leads to the distinction at macrolevel in characters of destruction of two composites. In the composite with $15 \%$ content of the hardening particles the macrocrack also has mainly vertical character. The mesocracks growing from the opposite edges of the specimen getting to a region of a dynamic influence and start to render the interference against each other that leads to their confluence at a final stage of deforming.

The particles flow characters in the composites are shown in the shift-fields. For example on the Fig. 9, 10 the shift-fields in composites related to the noted grain are presented for three consecutive times.

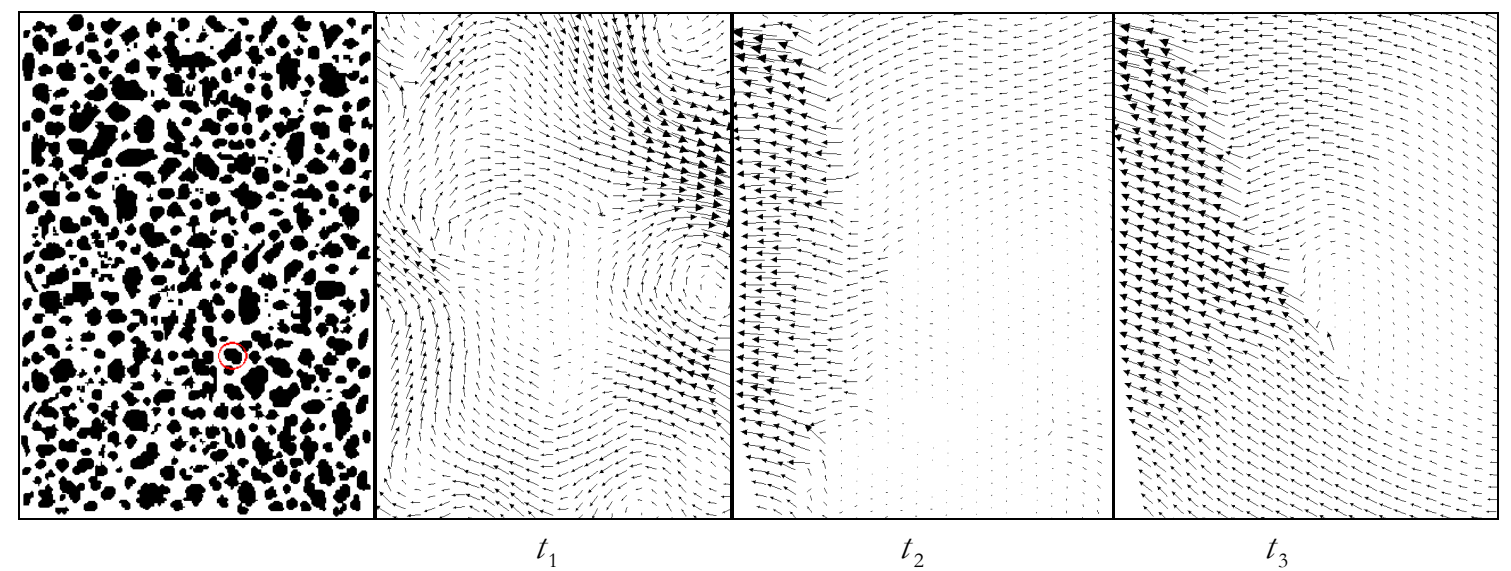

Figure 9: Simulation shift-fields for the composite with $40 \%$ content of the hardening particles in the case of ideal sliding on the loading border.

Shift-fields possess a strongly pronounced heterogeneity. The evolution of the shift-fields in time shows that the stressstrain state in the area of the fixed grain changes during the loading from compression on the incipient state of deforming then shears and formation of the curls and tension during the composite fragmentation. At main crack formation shiftfield shows the correlated locomotion of composite fragments in the normal direction to main crack. 

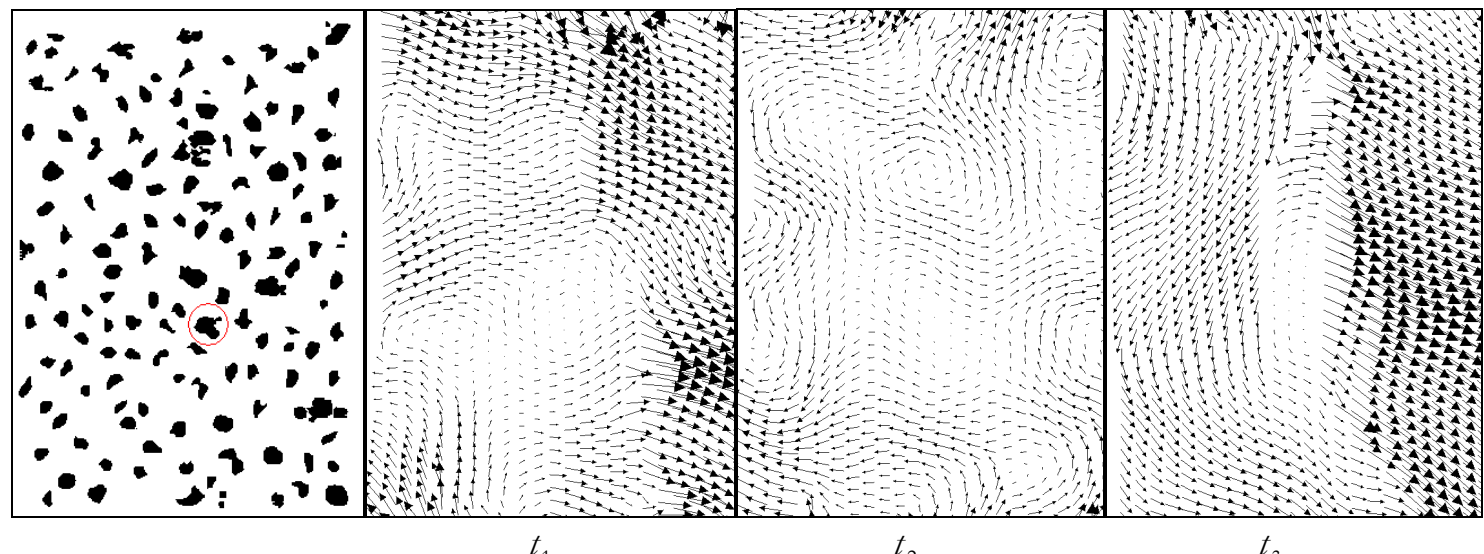

Figure 10: Simulation shift-fields for the composite with $15 \%$ content of the hardening particles in the case of friction on the loading border.
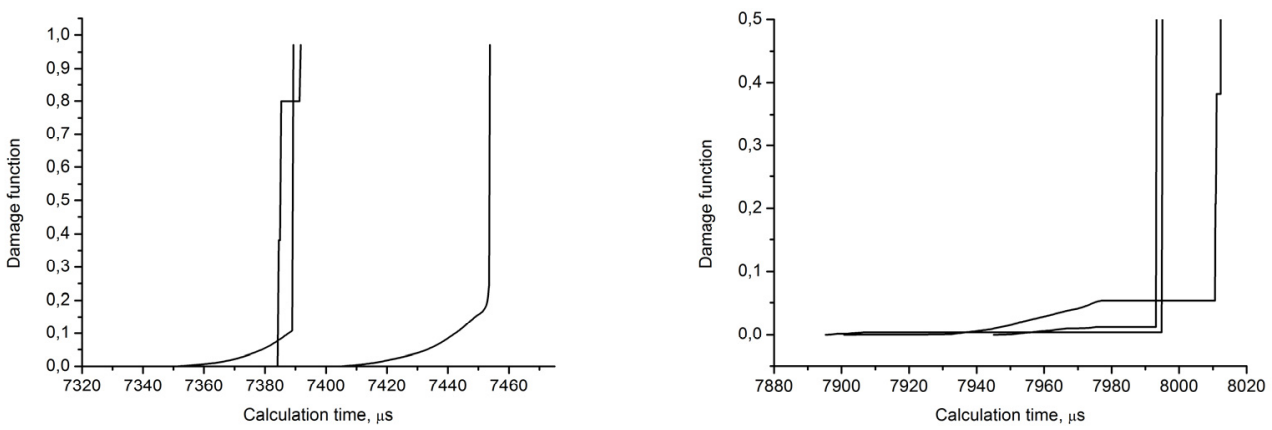

Figure 11: Damage function monitoring for several particles of the specimen which are in the region of a prospective dynamic influence in the composite with 15\% content of hardening particles ( $\mathrm{a}$ - in the case of friction on the loading border, $\mathrm{b}$ - in the case of ideal sliding on the loading border).

The pattern of damage function monitoring for several particles in the specimen volume located in the region of the prospective mutual dynamic influence (Fig. 11) shows the presence of slow quasistationary phase of damage growth and superfast phase of evolution - the blow-up regime with the damage growth in the particle of the composite to it's maximum value. From Fig. 11 also it is visible that damage in the particles consistently reaches the maximum value consecutively. The similar behavior can testify the migration of deformation activity. After the finish of the blow-up regime in one of the particles it begins in another (or the quasistationary phase in the next particle proceeds) and next the superfast phase of evolution occurs and there is a migration of deformation activity to the next particle.

\section{A generalization of the quasi-brittle medium on the case of $3 D$ simulation}

The developed model of quasibrittle medium was applied for the $3 \mathrm{D}$ numerical simulation of the brittle failure of ceramic composites. The 3D stochastic structure of the composite with 15\% content of the hardening particles with quasihomogeneous distribution was generated and presented on the Fig. 12. The same parameters as for the 2D simulation presented in Tab. 1 were applied in $3 \mathrm{D}$ simulation. The results of uniaxial compression at different boundary conditions are presented on the Fig. 13.

From the simulation patterns presented on the Fig. 13 we can see that the generalization of the developed model on the case of 3D simulation qualitatively gives the same results. Particularly we have the formation of the meso-cracks on the incipient stages of deforming mainly co-directed with the loading force. At later stages of deforming we can see that the formation of bridges between meso-cracks leads to the macro-crack formation which can be either co-directed with the loading force or disoriented to some angle with the loading force. In several cases the growing meso-crack change it's direction on the angle of $90^{\circ}$ when it gets into the region of the high compression near the hardening particle; such behavior at crack growing was observed in the experiments [23]. 


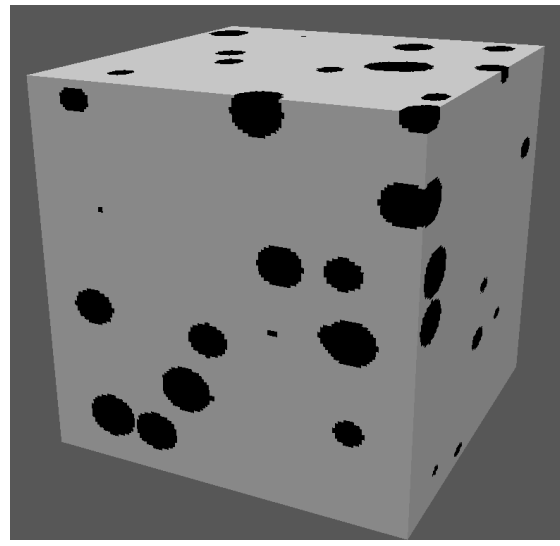

(a)

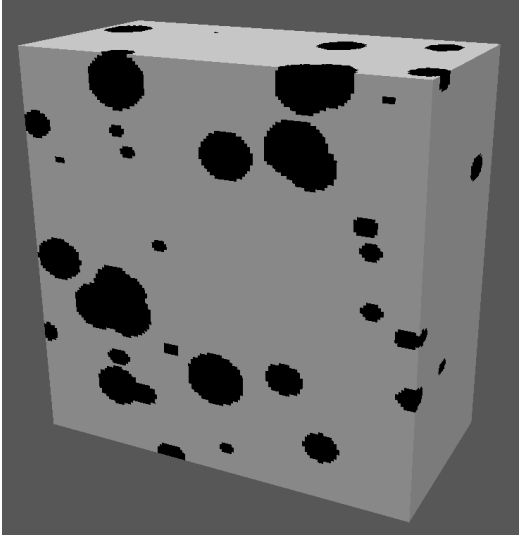

(b)

Figure 12: The 3D generated structural model of the ceramic composite with $15 \%$ contain of the hardening particles: the whole specimen (a) and a middle clip (b).

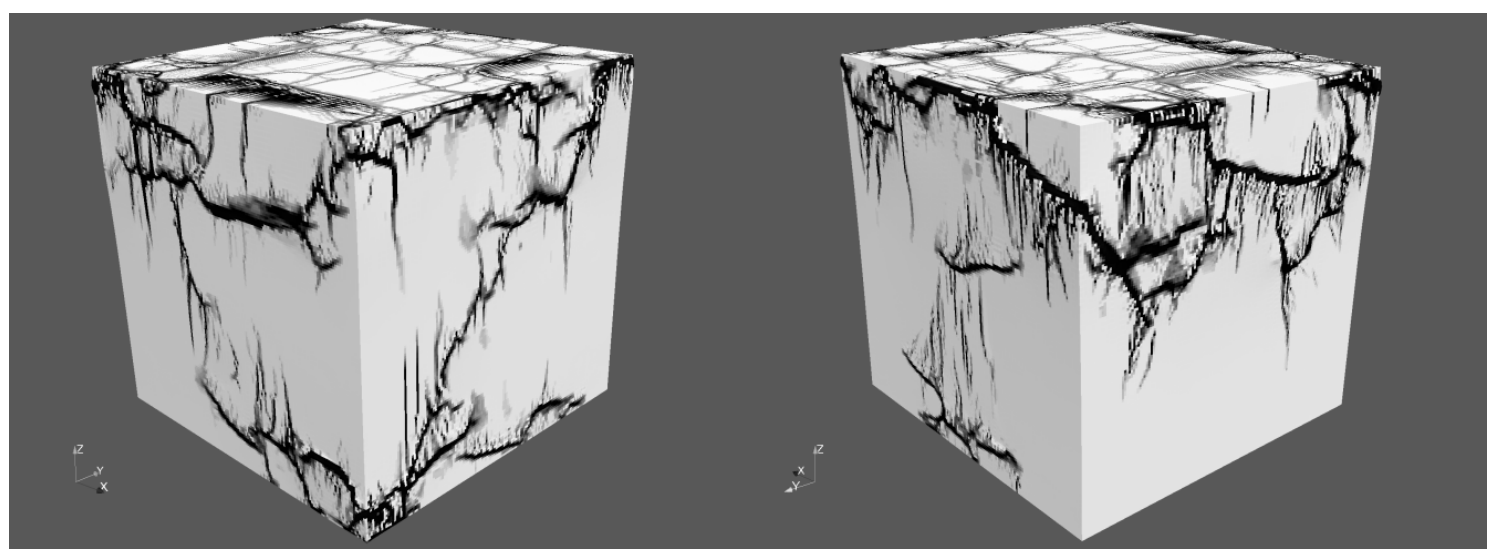

(a)

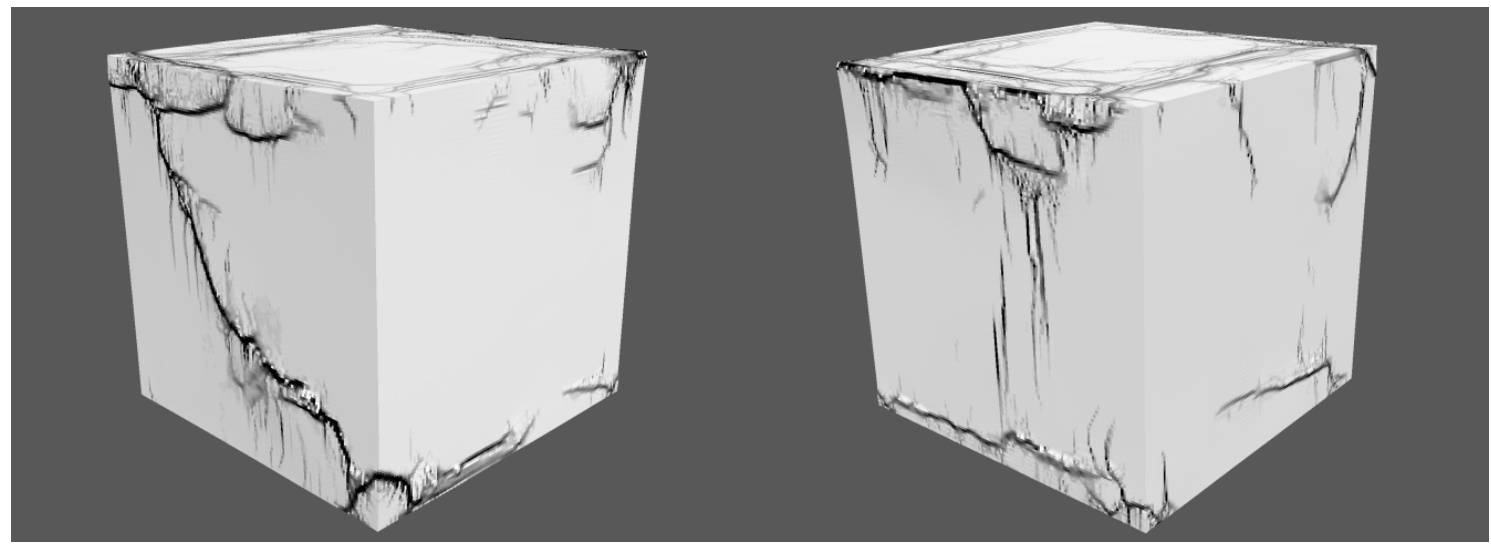

(b)

Figure 13: Simulation patterns: cracking of the ceramic composite with $15 \%$ content of hardening particles (2 views from opposite sides) at ideal sliding (a) and fixing (b) on the loading border. 


\section{CONCLUSIONS}

I $\mathrm{n}$ the considered model of medium the limiting condition in the loaded material is formed during loading and depends on the stressed state kind.

Studying the laws of brittle and quasibrittle failure of composite ceramic materials with the usage of the developed model of quasibrittle medium it was shown that failure process always educes in two stages - slow steady accumulation of inelastic deformations and damages in all hierarchy of structurally-scale levels as the quasistationary phase is replaced by the blow-up regime - the superfast catastrophic phase of evolution of system when the failure process reaches the macrolevel.

At ideal sliding on the loading border failure has a brittle character when the linear steepening of stresses is followed by the global loss of stability and degradation of elastic and strength properties of composites to zero. At friction on the loading border the stage of pre-failure inelastic deforming of the composites with formation of the local areas of the strength loss which occupies some percent of macrodeformation is observed. Thus the stage of evolution of system in the blow-up regime is tightened.

In the case of constrained deformation the other scenario of the composite evolution is observed. The resource of elastic and strength properties of medium is spent gradually. During the general evolution of the system in the blow-up regime the sequence of steepens and relaxations of stresses on the $\sigma-\varepsilon$ diagram is observed.

It is shown that brittle and quasibrittle failure of materials descends mainly in the tension stresses areas where the rate of elastic and strength properties degradation of medium is bigger on several orders. At pre-failure stage the confluence of the mesocracks in percolation net evolves in the macrocracking at the final stage of deforming.

\section{REFERENCES}

[1] V.E. Panin, A.D. Korotaev, P.V. Makarov, V.M. Kuznetsov, Izvestiya of the High schools. Physics, 9 (1998) 8.

[2] P.V. Makarov, Phys. Mesomech, 11 (3) (2008) 19.

[3] S.P. Kurdyumov, Blow-up regimes. Idea evolution, Ed. G.G. Malinetsky, $2^{\text {nd }}$ ed., Fizmatlit, (2006) 312.

[4] P.V. Makarov, Phys. Mesomech, 13(5) (2010) 97.

[5] V.Z. Parton, E. M. Morozov, Mechanics of elasto-plastic failure, Science, (1974) 416.

[6] P.V. Makarov, Geology and geophysics, 48(7) (2007) 724.

[7] P. V. Makarov, I. Yu. Smolin, Yu. P. Stefanov, Nonlinear mechanics of geomaterials and geomedia, Novosibirsk, SB RAS Geo, (2007) 235.

[8] P.V. Makarov, Phys. Mesomech, 8 (6) (2005) 39.

[9] E. P. Evtushenko, M. O. Eremin, Yu. A. Kostandov, P.V. Makarov et alii, Phys. Mesomech, 15(3) (2012) 35.

[10] I. A. Garagash, V. N. Nikolaevskiy, Successes of mechanics, 12(1) (1989) 131.

[11] S. N. Zhurkov, B. N. Narzulaev, ZhTF, XXIII(10) (1953) 1677.

[12] A.V. Stepanov, Basics of the practical crystal strength, Science (1974) 131.

[13] V. R. Regel, A. I. Slutzker, E. E. Tomashevskiy, Science, (1974) 560.

[14] I.I. Frenkel, Introduction to the metals theory, Fizmatlit, (1958) 424.

[15] R. Becker, Physikalische zeitschrift, 25(7) (1925) 919.

[16] E. J. Orowan, The creep of metals, West Scot. Iron and steel Inst., 54 (1947) 45.

[17] M. L. Wilkins, Computer simulation of dynamic phenomena, Heidelberg, Springer, (1999) 265.

[18] G. I. Barenblatt, G.P. Cherepanov, Izv. Of USSR AS, Mechanics and Mechanical engineering, 3 (1960).

[19] Yu. P. Stefanov, Phys. Mesomech, 8(3) (2005) 129.

[20] Yu. A. Kostandov, P. V. Makarov, M. O. Eremin, I. Yu. Smolin, I. E. Shipovskii, International Applied Mechanics, 49 (1) (2013) 95.

[21] B. Paliwal, K. T. Ramesh, J. of the Mechanics and Physics of Solids, 56 (2008) 896.

[22] S. V. Panin, P. S. Lyubutin, S. P.Buyakova, S. N. Kulkov, Phys. Mesomech, 11(6) (2008) 77.

[23] J. D. Kuntz, G. D. Zhan, A. K. Mukherjee, MRS Bull., 29 (2004) 22. 